\title{
Evaluation of velocity fields in horizontal gas-liquid intermittent flows using Stereoscopic-PIV and instantaneous masking procedure
}

\author{
L. S. Fernandes ${ }^{1}$, R. S. N. de Mesquita $^{1}$, F. J. W. A. Martins ${ }^{1,2}$, L. F. A. \\ Azevedo ${ }^{1, *}$ \\ ${ }^{1}$ Mechanical Engineering Department, Pontifical Catholic University of Rio de Janeiro, Brazil \\ ${ }^{2}$ Current address: Institute for Combustion and Gas Dynamics - Tomography Group, University of \\ Duisburg-Essen, Germany \\ *Correspondent author: Lfaa@puc-rio.br
}

\begin{abstract}
The main goal of this work was to obtain well-converged liquid velocity profiles for intermittent gas-liquid flows in a horizontal pipe. To this end, air and water with superficial velocities of $J_{G}=0.5 \mathrm{~m} / \mathrm{s}$ and $J_{L}=$ $0.3,0.4$ and $0.5 \mathrm{~m} / \mathrm{s}$, respectively, were driven into a $18-\mathrm{m}$ acrylic test section with an inner diameter of $40 \mathrm{~mm}$. All three-components of the velocity vectors were measured in a pipe cross-section using a highfrequency stereoscopic PIV system, together with the laser induced fluorescence technique. Photogates were used to measure the unit cell translational velocity, as well as to trigger data acquisition, allowing the calculation of ensemble-averaged velocity fields at specific positions, referenced to the gas-bubble nose tip position. An instantaneous image masking procedure was implemented, allowing the determination of non-dimensional ensemble-averaged velocity profile in the liquid film, referenced to gas-bubble boundary. The high-frequency system employed allowed the determination of the influence of the faster-moving gas bubble on the liquid velocity field in the plug region. The data presented are relevant to the validation and improvement of one-dimensional two-phase numerical models, as well as to better understand this complex flow.
\end{abstract}

\section{Introduction}

Two-phase gas-liquid flows can be found in many engineering applications, ranging from oil and gas transport and production lines to nuclear reactor cooling systems. In fact, the continuously search for new oil reservoirs at deeper offshore locations has led to an increase in the distance from oil pre-processing facilities to well-heads (Hua et al., 2012), increasing temperature and pressure variation along the production lines and enhancing, therefore, the possibility of occurrence of different gas-liquid flow patterns.

There are two main reasons why intermittent flow in a horizontal pipe might occur. First, due to accumulation of liquid at lower parts of pipelines through hilly terrains, what is called severe slugging (Al-Safran et al. (2005). Second, due to the natural growth of waves, due to Kelvin-Helmotz instability phenomenon in a stratified flow (Taitel and Dukler, 1976). In fact, an analysis of classical gas-liquid flow pattern maps (Baker. 1953; Brennen, 2005) clearly shows that the intermittent flow regime (elongated bubble flow and slug flow) occupies a large area in the maps, i.e., it is the dominant flow pattern for a considerable combination of gas and liquid flow rates, justifying the study of this gas-liquid flow pattern.

The horizontal intermittent flow is usually characterized by a succession of unit cells, each one being composed of two regions, namely, liquid plug and liquid film. In the liquid film region, gas flows at a higher velocity at the upper part of the pipe, while slower liquid flows at the bottom part. Differently, in the liquid plug region, the liquid phase occupies the whole pipe cross section. The presence of dispersed bubbles in the liquid plug is a condition used by many authors to distinguish between the elongated-bubble flow and the slug-flow regimes. In the present work, such differentiation will not be considered.

The transient intrinsic characteristic of the intermittent flow described above causes abrupt and large variation in the fluid density at a specific location, what can generate severe damage to both equipments or 
structures (Fabre et al., 1990). A good understanding and modelling of the intermittent flow is, therefore, fundamental to the proper operation and design of the flow systems. This work provides well-converged velocity profiles at different locations of the unit cell that can be used not only to improve and validate numerical simulations, but also to help elucidate the physic mechanisms governing this complex phenomenon.

\section{Experiment and data evaluation}

Figure 1 shows a schematic view of the test section. It consisted of a $18-\mathrm{m}$ long acrylic pipe with an internal diameter $\mathrm{D}$ of $40 \mathrm{~mm}$. Air and water were driven into the test section by 2 centrifugal compressors assembled in series and by a progressive cavity pump, respectively. This specific pump was used because it provides a flow rate independent of the outlet pressure, eliminating possible liquid flow rate variations due to pressure oscillations. Both fluids entered the test section through a Y-junction. The flow developed through a pipe length of $14.8 \mathrm{~m}(370 \mathrm{D})$ upstream of the measurement region, which was located $6.4 \mathrm{~m}$ downstream of a $180^{\circ}$ horizontal curve and $3.6 \mathrm{~m}(90 \mathrm{D})$ upstream of the end of the horizontal pipe. Water and air calibrated rotameters were used to measure the liquid and gas flow rates.

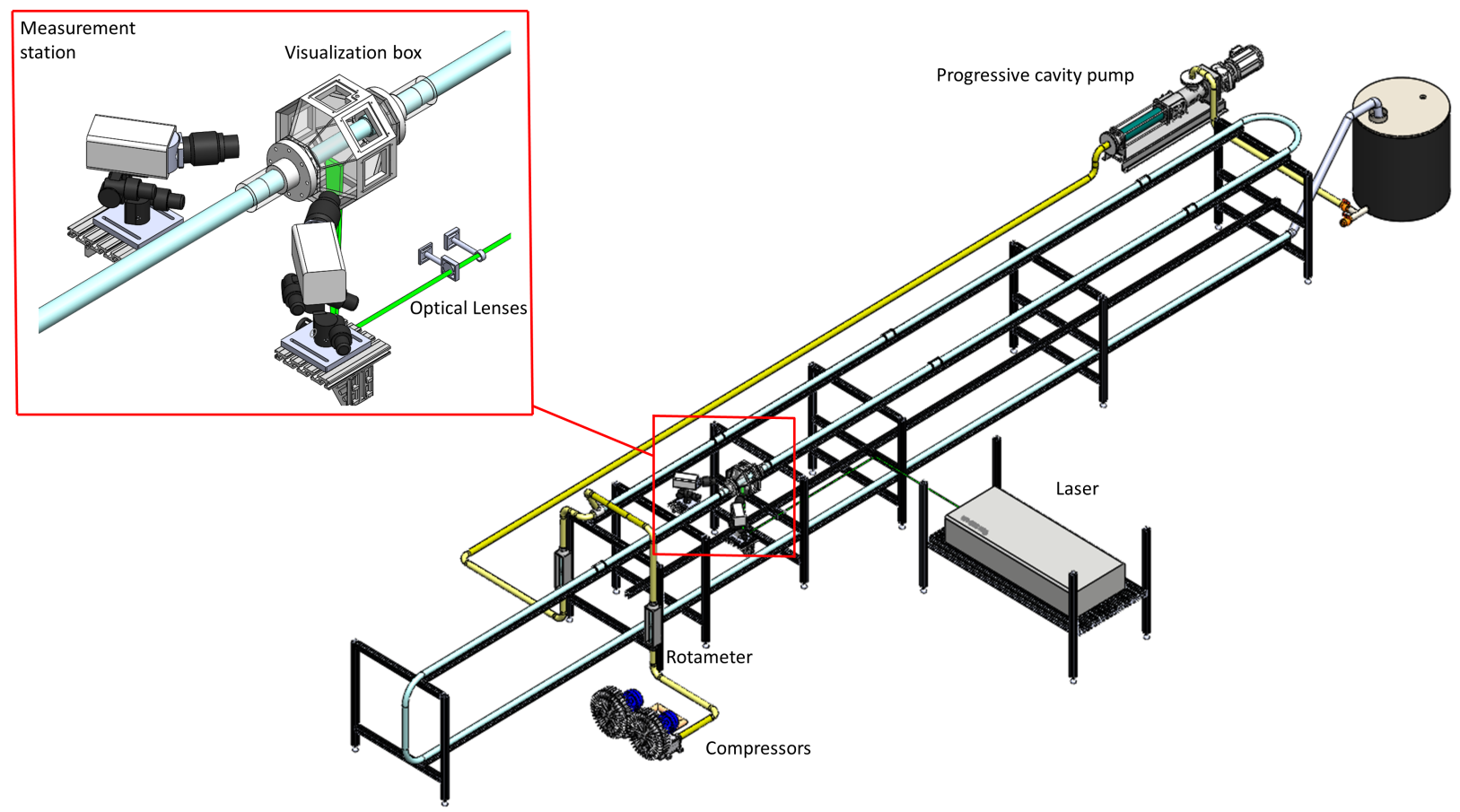

Figure 1: Schematic view of the test section, highlighting the measurement station

A stereoscopic-PIV system (SPIV) was used to measure all three-components of the velocity vector in a pipe cross-sectional plane. A 2-mm-thick laser light sheet was provided by a Litron LDY304 pulsed laser (2x30 mJ @ 400Hz), while two high-speed CMOS Phantom M340 cameras (1280x1280 pixels @ 400Hz) were symmetrically mounted at opposite sides of the pipe, in a horizontal arrangement, as indicated in figure 1. The cameras were placed downstream the laser light sheet, in order to reduce gas-bubble obstruction of the film region (Fernandes et al., 2018). The 105-mm Nikon-Nikkor lenses with $\mathrm{f} \#=11$ aperture were tilted in relation to the camera sensor using Scheimpflug adaptors. A visualization box, filled with water, was used to minimize optical distortions due to the pipe curvature. Also, in order to mitigate optical distortions, the pipe wall thickness at the measurement region was machined down to $1 \mathrm{~mm}$. Both cameras and laser were controlled by a TSI model 610036 synchronizer.

A set of three equally-spaced infra-red photogates were positioned upstream of the measurement region. The differences in the refraction indexes of water and air deviated the infra-red beam away from the photogate detector when water was present, allowing the determination of the phase flowing in the upper part of the pipe. The first two photogates were used to measure the translational velocity of the gas-bubble, 
which was assumed to be the translational velocity of the unit cell. The third photogate was used to trigger the image acquisition. The same photogate system was used with success in previous works of the group (De Oliveira et al., 2015; Fernandes et al., 2018).

In gas-liquid PIV measurements, the interface is often a critical region. The light scattered at the interface can not only obscure particles near those regions, but also damage the camera sensors due to excess of light. A common practice to avoid such problem is to use the laser-induced fluorescence technique (LIF) (Lindken and Merzkirch, 2002). In this work, polystyrene fluorescent particles impregnated with Rhodamine $6 \mathrm{G}$, with diameter ranging from 1 to $20 \mu \mathrm{m}$ and density of $1.19 \mathrm{~g} / \mathrm{cm}^{3}$ were used as seeding material. Highpass optical filters were installed in front of the camera lenses, in order to transmite the light emitted by the particles and block the laser light scattered at the interface.

\subsection{Camera calibration}

The cameras were calibrated using a specially designed calibration rig. It consisted of a matrix of 140 equally-spaced points which was printed on a transparent sheet and glued on the surface of a propylene disk, as can be seen in figure 2(a). The disk was connected by a rod to a micrometer head, with a resolution of $10 \mu \mathrm{m}$, allowing the axial translation of the target, as shown in figure 2(b). The rod passed through an aperture in a cylindrical sleeve with rubber O-rings, which fitted the external part of the acrylic pipe, properly sealing it.

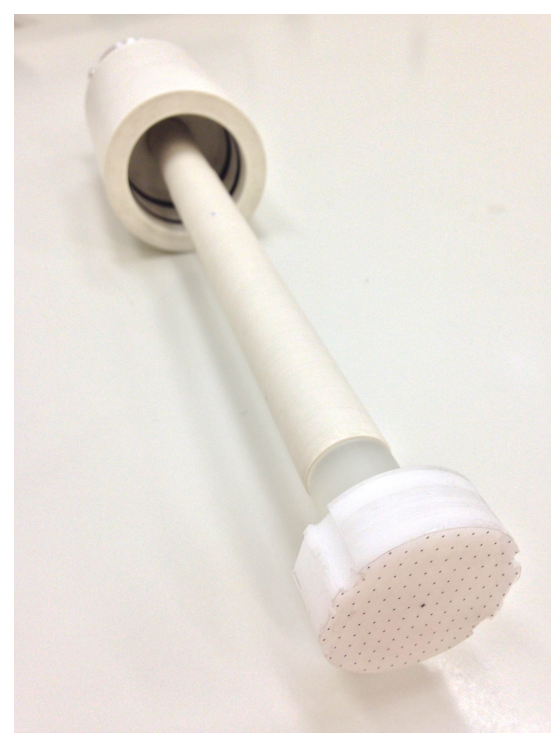

(a)

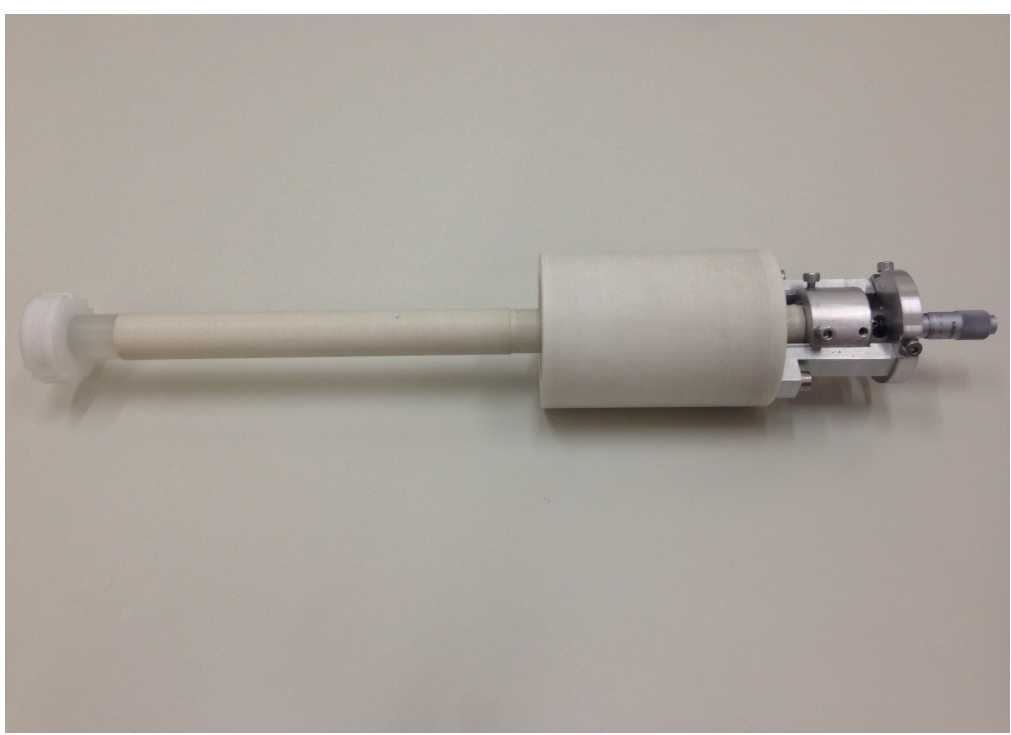

(b)

Figure 2: Different views of the calibration target.

During the calibration procedure, the pipe downstream of the visualization box was removed, the target inserted into the measurement region and the upstream pipe filled with water. A total of three calibration images of the planar target, equally-spaced of $0.5 \mathrm{~mm}$ along the pipe axis and centered at the laser light sheet position were acquired for each camera. A calibration polynomial of third order in $\mathrm{x}$ and $\mathrm{y}$ (inplane coordinates) and second order in $\mathrm{z}$ (out of plane coordinate) was generated based on the target points positions. A self-calibration procedure (Wieneke, 2005) using low-density particle images was employed to refine the calibration polynomia that were used both to dewarp the acquired images and later to reconstruct the three-component velocity vectors.

\subsection{Experimental procedure}

The infrared photogate system described in section 2 was used to trigger the image acquisition. When the third photogate detected the passage of the gas bubble-nose, it automatically started the acquisition of 220 image pairs, for each camera, at a frequency of $400 \mathrm{~Hz}$, covering the passage of the gas-bubble nose through 
the measurement region. Small manual adjustments were performed to determinate in which image of the set of time-series data, the bubble nose was located. Whenever there were at least 80 image pairs registering the liquid film and the liquid plug (i.e., gas bubble nose tip between images 81 and 140 of the time-series), the run was validated. This procedure was repeated 1500 times, and image pairs were separated according to the time-distance from the gas-bubble nose (images captured at $2.5 \mathrm{~ms}, 5.0 \mathrm{~ms}$, etc after or before the passage of the bubble-nose). The measured bubble-translational velocity, here considered as the unit cell translational velocity, was used to transform the time between consecutive image samples into spatial distance, following the same procedure of Fernandes et al. (2018).

Three different cases were measured, with gas and liquid superficial velocities of $J_{G}=0.5 \mathrm{~m} / \mathrm{s}$ and $J_{L}=$ $0.3,0.4$ and $0.5 \mathrm{~m} / \mathrm{s}$ respectively, as summarized in table 11 . The mixture Reynolds number was calculated based on the mixture superficial velocity and pipe diameter.

Table 1: Summary of the cases measured

\begin{tabular}{ccccc}
\hline Case & $\begin{array}{c}J_{L} \\
(\mathrm{~m} / \mathrm{s})\end{array}$ & $\begin{array}{c}J_{G} \\
(\mathrm{~m} / \mathrm{s})\end{array}$ & $\begin{array}{c}J_{m} \\
(\mathrm{~m} / \mathrm{s})\end{array}$ & $\begin{array}{c}\text { Reynolds } \\
(\text { mixture })\end{array}$ \\
\hline $\mathbf{1}$ & 0.3 & 0.5 & 0.8 & 32000 \\
$\mathbf{2}$ & 0.4 & 0.5 & 0.9 & 36000 \\
$\mathbf{3}$ & 0.5 & 0.5 & 1.0 & 40000 \\
\hline
\end{tabular}

Images were pre-processed by subtracting the average image intensity, in order to minimize the influence of the background during images processing. Cross-correlation was performed on the dewarped images, using a recursive grid with final interrogation window of $32 \times 32$ pixels, with $50 \%$ overlap. The final measured velocity vector field had over 3700 vectors, in the liquid phase, equally spaced of $0.6 \mathrm{~mm}$. All image pairs were processed using the software Insight 4G, by TSI.

\subsection{Masking procedure}

PIV evaluation relies on the measurement of the motion of tracer particles that are seeded in the flow. In the present work, the water was seeded, but not the gas, so the PIV processing was restricted to liquid regions containing particles. Fernandes et al. (2018) applied algorithmic masks based on the maximum and minimum pixel intensities of the particle images of the left and right cameras for each cross-sectional position, in relation to the bubble nose tip. The same local algorithmic mask was used for all PIV evaluations at a particular cross-sectional location. This was possible because the gas-bubbles were relatively wellbehaved, due to the laminar characteristic of the liquid flow composed of a high-viscosity water-glycerin solution. In the present work, however, water was used as the liquid phase, leading to a turbulent intermittent flow with stochastic gas-bubble shapes (De Oliveira et al., 2015). The region occupied by the gas phase, in the same pipe cross section relative to the detected nose position tip, varied significantly when comparing different instantaneous particle images, although the gas bubbles were always in the upper part of the pipe. This effect was more pronounced for images in the close vicinity of the bubble nose.

The regions containing particles were automatically determined for each instantaneous image pair in each cross-sectional location, employing instantaneous algorithmic masks. The algorithmic masks were created based on the information of the presence, or lack, of particles within the interrogation windows as follows. First, instantaneous dewarped particle images from the first and second laser pulses, after background subtraction, were combined to artificially increase the particle concentration. As a second step, a sliding standard deviation filter with a window of 11x11 pixels was applied to detect particles. This procedure is robust against possible shot-to-shot fluctuations of the laser. Next, instantaneous binary masks were created by thresholding those filtered images from each camera. Threshold values for the left and right camera images were selected based on the average intensity level outside the pipe (dark region). The binarized images were composed of regions of "ones", related to medium- and high-intensity particles, and others of "zeros", mostly related to noise and low-intensity particles. As a fourth step, the binary mask was divided into $32 \times 32$ pixel regions with centers coinciding with those that would form the final vector grid. If a particular region contained any bright particle, the entire 32x32 pixel region was set to "one", otherwise the region was set to zero. Following, dilate and erode binary operations (Gonzales and Woods, 2008) were used to fill up possible holes and to connect regions. This was necessary due to the conservative higher threshold levels employed that did not detect the low-intensity particles. The instantaneous masks for the left and right cameras correctly represented the seeded regions (non-obstructed liquid phase) what was verified by 
visual inspection of several image samples. Finally, the final instantaneous mask at each cross-section was obtained from the minimum between the left and right masks ("AND" logical operator). It is important to mention that the mask regions with ones were always equal or smaller than the liquid regions due to possible viewing obstruction caused by the bubble nose passage, as explained in Fernandes et al. (2018). Figure 3 shows a typical instantaneous masking procedure, combining the image obtained from the left an the right cameras, at an specific position relative to the gas bubble nose tip. Additionally, instantaneous binary masks representing the gas phase at each cross-section were estimated based on the maximum between the left and right masks ("OR" logical operator), following Fernandes et al. (2018).

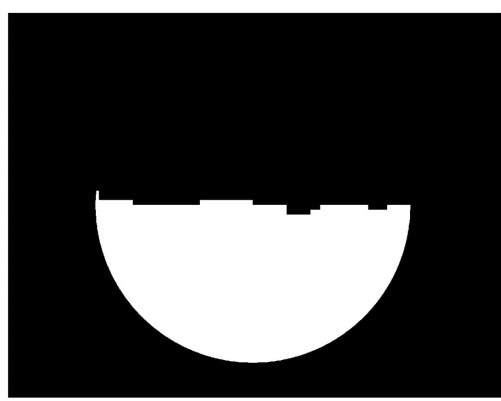

Left Camera

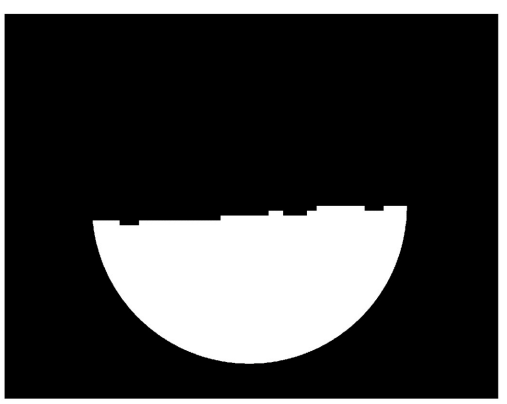

Merged Image

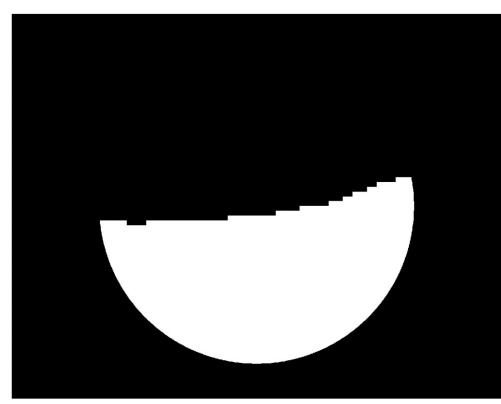

Right Camera

Figure 3: Masking procedure for a specific instantaneous image in the liquid film. The merged image represents the instantaneous algorithmic mask for PIV processing at this particular cross-section, and it was generated by the minimum between the binary masks of the left and right camera images.

The ensemble-averaged velocity fields were computed only from valid instantaneous vectors. That means, vectors within the instantaneous masks and within 3 local standard deviation from their neighbours (Westerweel and Scarano, 2005). The final ensembled mask at each cross section, representing the nonobstructed liquid phase, was composed by the grid positions where at least $68.3 \%$ of the total amount of vectors available for the determination of the the ensemble-average (1500 samples) were not masked out by the instantaneous masks. This represents a conservative approach corresponding to a coverage of one standard deviation for a normal distribution.

\section{Results}

\subsection{Velocity profiles in the liquid plug region}

Figure 4 presents the measured streamwise velocity profiles in the liquid plug region for the three cases investigated. The profiles were extracted from the measured ensemble averaged velocity fields at a vertical line along the pipe diameter. Also shown in the figure is a schematic view of the slug flow unit cell, indicating the positions of the vertical profiles in relation to the gas-bubble nose. As can be seen, the faster-moving gas-bubble deforms the velocity profiles at positions close to the bubble-nose (figures $4 \mathrm{a}$ and $\mathrm{b}$ ), creating two local maximum points, located at approximately $y / R=-0.1$ and $y / R=0.5$ and a local minimum point, located at $y / R=0.1$. The maximum velocity is located just downstream of the gas-bubble nose tip, approximately at $y / R=0.5$. This effect is more evident at higher superficial velocities (Case 3), where a higher translational velocity of the gas bubble prevails (Hurlburt and Hanratty, 2002).

At farther positions inside the liquid plug (figures $4 \mathrm{k}$ and $\mathrm{d}$ ), the velocity profile resembles the format of a fully-developed turbulent velocity profile in a straight pipe. The vertical profiles are, however, not entirely symmetric, what can be associated to the presence of small dispersed bubbles at the upper part of the pipe.

The similarity between the streamwise velocity profiles at $2.35 \mathrm{D}$ and $6.2 \mathrm{D}$ inside the liquid plug raises a question whether there is a distance downstream to the gas-bubble nose where the velocity fields no longer change. To answer this question, the root mean square deviation (RMSD) between the velocity field located at 6.2 D downstream of the gas-bubble nose tip (figure $4 \mathrm{~d}$ ) and velocity fields at closer locations to the gas-bubble nose tip was calculated and presented in figure 5. As can be observed, after a distance of 

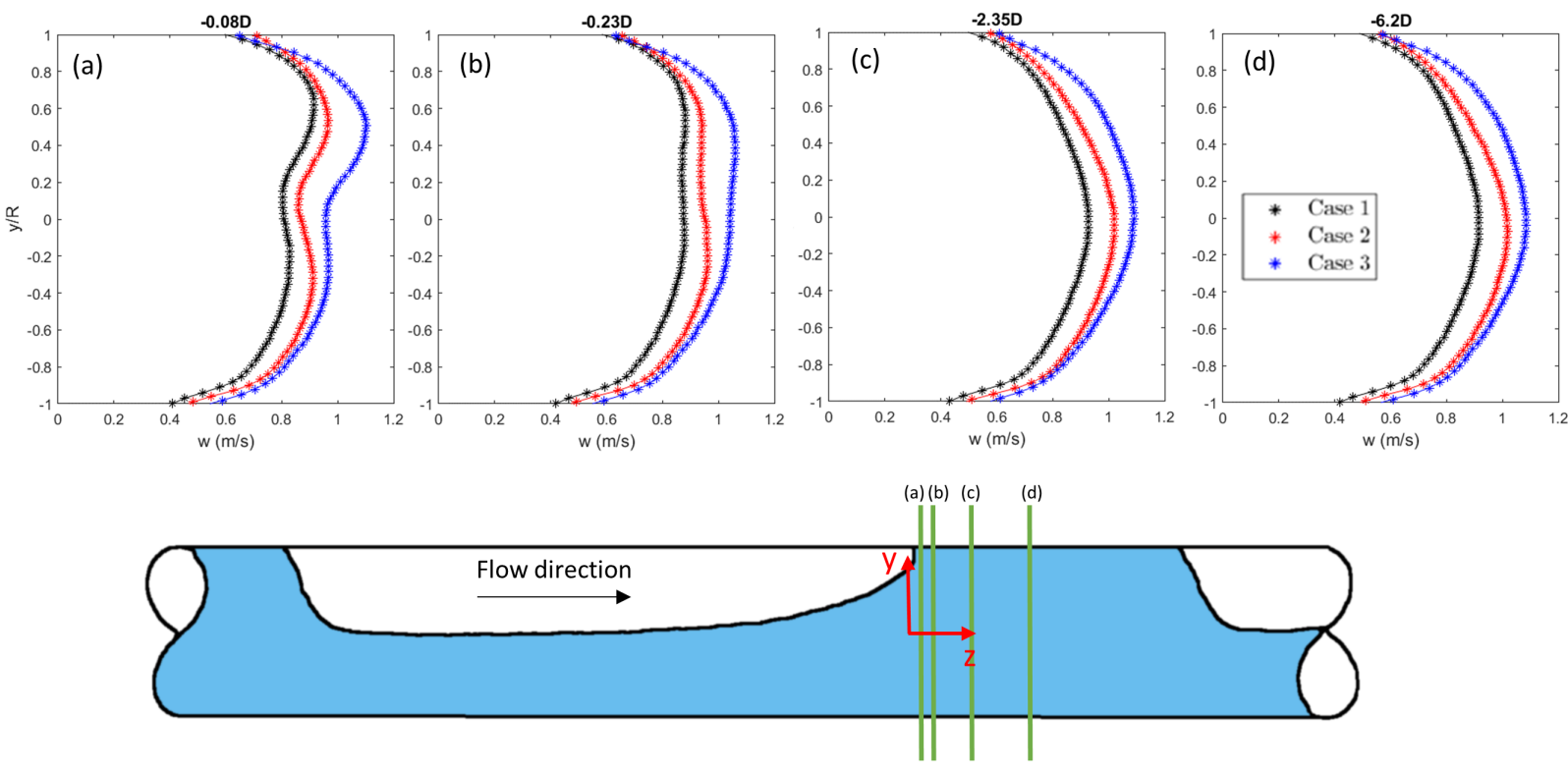

Figure 4: Streamwise velocity profiles at different locations inside the liquid plug for all 3 measured cases

approximately $0.8 \mathrm{D}$ from the gas-bubble nose, there are only minor variations in the streamwise velocity fields. The most significant changes happen less than 1 diameter downstream of the gas bubble nose.

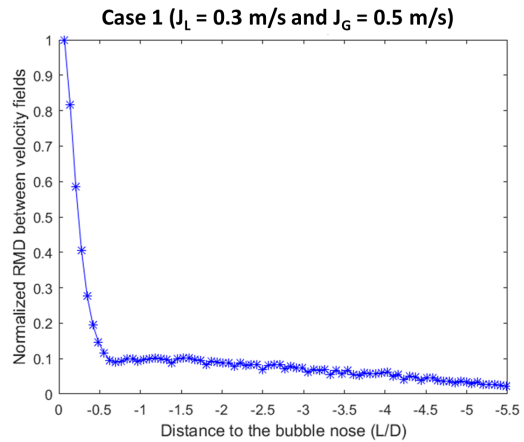

(a)

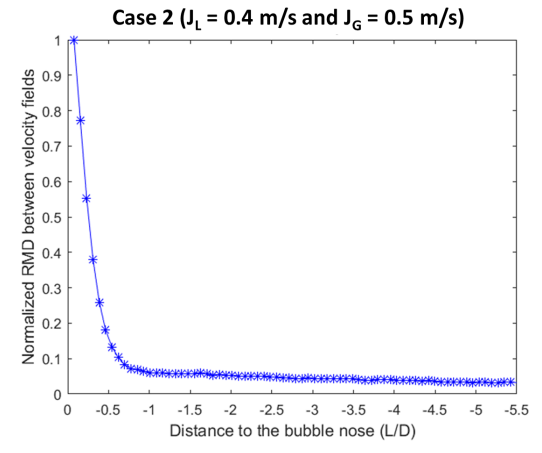

(b)

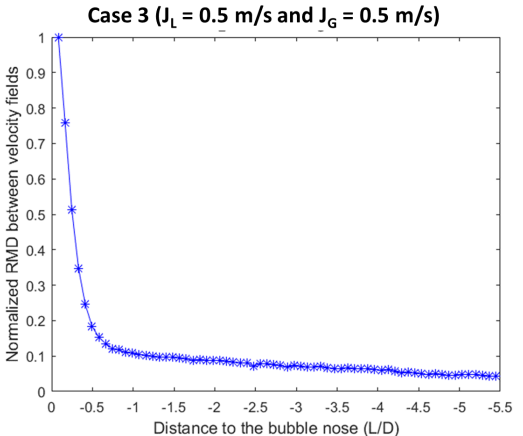

(c)

Figure 5: Normalized root mean square deviation between averaged streamwise velocity fields at different locations inside the liquid plug and the streamwise velocity field at $6.2 \mathrm{D}$ from the gas-bubble nose tip.

During the experiment, the superficial velocities of gas and liquid for each case remained constant. However, small oscillations in pressure can cause variations in the inlet flow rate of gas, given the fact that it was supplied by centrifugal compressors. A verification test can be made by calculating the mixture velocity. For incompressible flow, continuity dictates that the total flow rate and, therefore, the mixture velocity remains constant at any cross-section in the unit cell (Woods and Hanratty, 1999). Table 2 present the mixture velocity calculated by integrating the measured streamwise velocity fields in the liquid plug, for each case. The maximum deviation observed was of $3.6 \%$, for case 3 .

\subsection{Velocity fields in the bubble-nose region}

In this section, the behaviour of velocity fields of the averaged streamwise velocity in the bubble-nose region are evaluated. The averaged mask described above was used to separate gas regions, where seeding particles 
Table 2: Comparison of expected and measured mixture velocity for each case.

\begin{tabular}{cccc}
\hline Case & $\begin{array}{c}\text { Expected mixture } \\
\text { velocity }(\mathrm{m} / \mathrm{s})\end{array}$ & $\begin{array}{c}\text { Measured mixture } \\
\text { velocity }(\mathrm{m} / \mathrm{s})\end{array}$ & Error $(\%)$ \\
\hline $\mathbf{1}$ & 0.8 & 0.81 & $1.65 \%$ \\
$\mathbf{2}$ & 0.9 & 0.90 & $0.22 \%$ \\
$\mathbf{3}$ & 1.0 & 0.96 & $3.6 \%$ \\
\hline
\end{tabular}

were not presented, from the liquid regions, where PIV evaluation was performed.

Figure 6 present vertical liquid velocity profiles obtained from case 2 . It clearly shows the influence of the faster-moving gas-bubble, deforming the velocity profile at the upper part of the pipe, for positions downstream of the bubble nose tip, as already mentioned. An interesting feature presented here is the change in the velocity profile at positions around $y / R=0.4$ from the position just upstream of the gas-bubble nose $(0.08 \mathrm{D})$ to positions deeper inside the liquid film $(0.39 \mathrm{D}$ and $0.62 \mathrm{D})$. It seems that the gas-liquid interface moves at a lower velocity than that of the gas-bubble, causing a reduction on the streamwise velocity profile due to the non-slip condition on the gas-liquid interface. This issue will be better addressed in the next section.

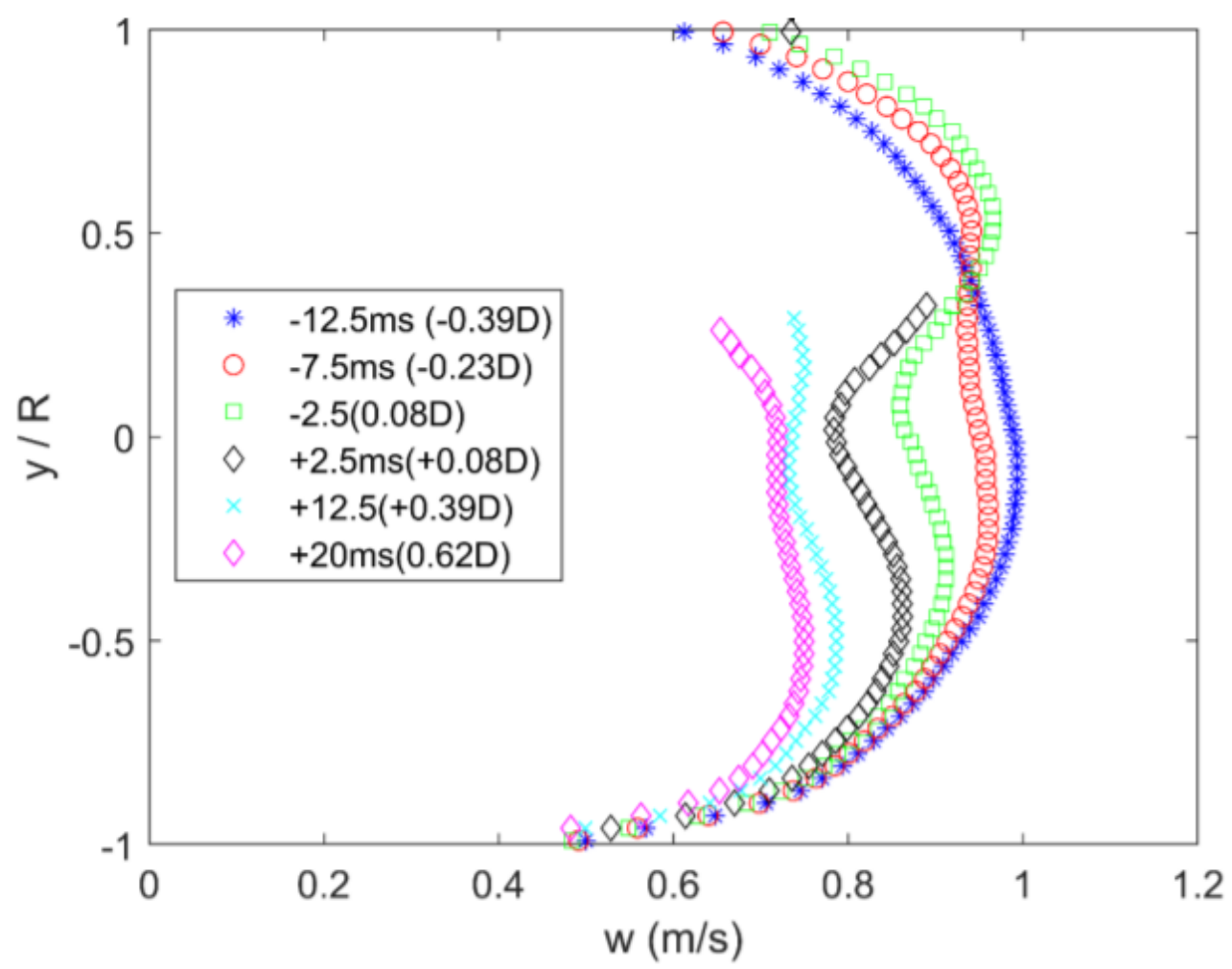

Figure 6: Averaged streamwise velocity profiles at different locations in the near region of the bubble nose for case $2\left(J_{G}=0.5 \mathrm{~m} / \mathrm{s}\right.$ and $\left.J_{L}=0.4 \mathrm{~m} / \mathrm{s}\right)$.

Figure 7 presents a schematic view of the slug unit cell, showing the averaged velocity vector fields in the vicinity of the gas bubble nose. Colormaps represent the streamwise component, while in-plane velocity is represented by vector arrows. As one can see, the faster-moving gas bubble not only pushes forward the downstream liquid (revisit figure 6), but it also pushes the liquid downwards (figure $7 \mathrm{a}$ and $\mathrm{b}$ ). This is the shedding mechanism, in which, for a frame of reference moving with the unit cell, liquid from the liquid plug is picked up by the film close to the gas-bubble nose tip, compensating the liquid lost by the film near the gas bubble tail, to the upstream liquid plug.

For the first measurement planes just upstream of the gas-bubble nose (figure $7 \mathrm{k}$ and d), a circular motion 
around the gas-bubble is formed, where liquid is pushed away from regions that will be occupied by the gas bubble. Further inside the liquid film, the downwards movement dominates the in-plane motion, what result in the formation of two counter-rotating vortices, as seen in figure $7 \mathrm{~d}$.

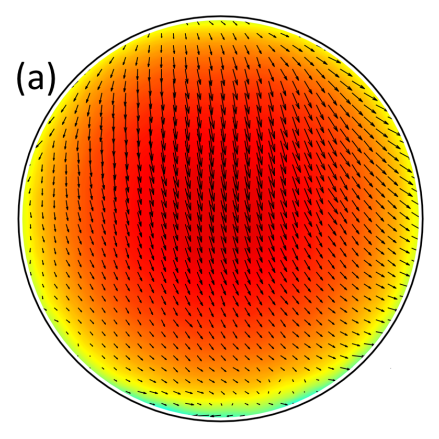

(b)
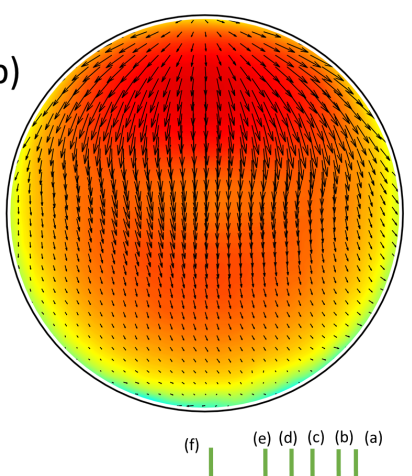

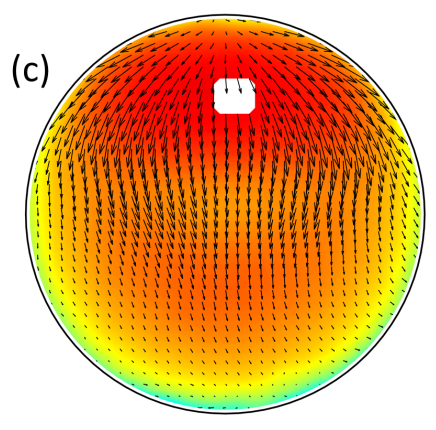

$\mathrm{w}(\mathrm{m} / \mathrm{s})$

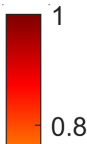

0.8

0.6

0.4

(d)
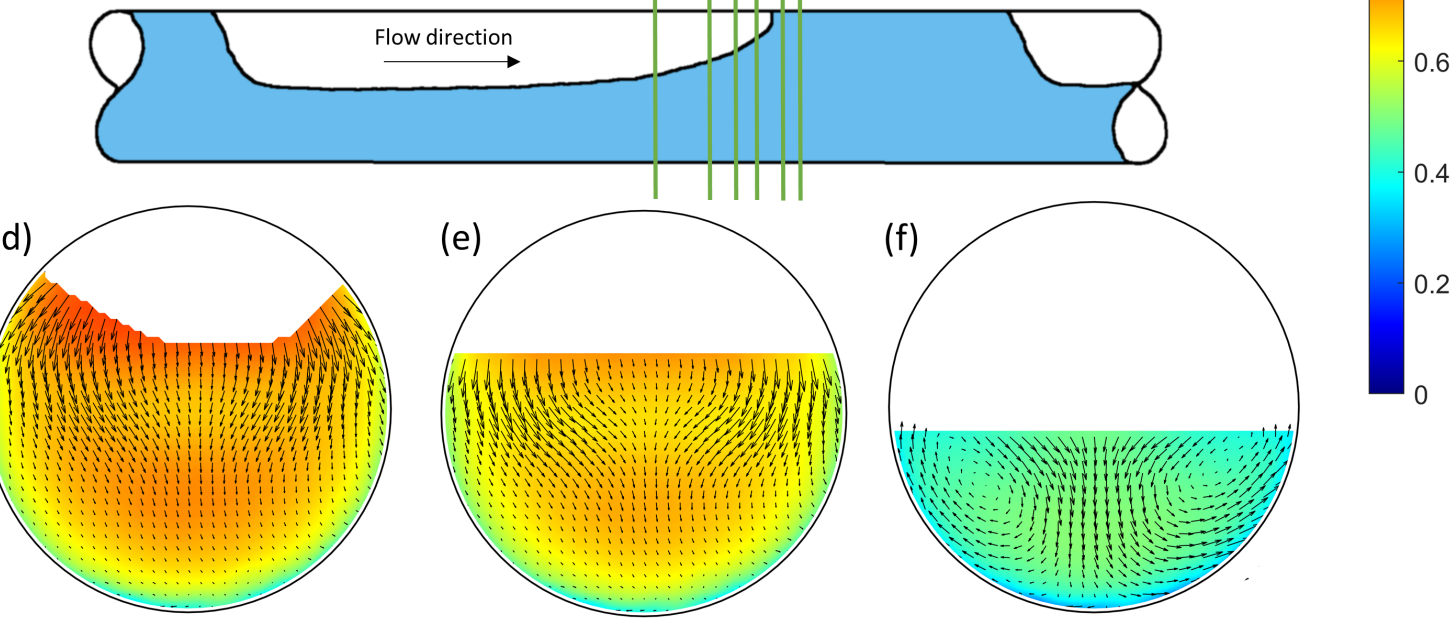

Figure 7: Averaged velocity fields at different locations in the region near the bubble nose for case $2\left(J_{G}=\right.$ $0.5 \mathrm{~m} / \mathrm{s}$ and $J_{L}=0.4 \mathrm{~m} / \mathrm{s}$ ). Colormaps represent the averaged streamwise velocity component, while in-plane components are represented by vector arrows.

\subsection{Liquid film region}

Figure 8 presents vertical profiles of the streamwise velocity at different positions inside the liquid film region. As expected, the mean velocity increases from case 1 to case 3, given the increase in the mixture velocity. Another interesting effect is the decrease in the mean velocity when moving farther into the liquid film, far from the bubble nose. The same behaviour was also observed for laminar intermittent flows by Fernandes et al. (2018). The velocity profiles were computed from the averaged streamwise velocity fields using the mean liquid mask from the non-obstructed liquid regions described in section 2.3.

An analysis of figure 8 indicates that the upper part of the liquid film is moving at a smaller velocity than the liquid mean velocity (i.e., there is a concavity in the liquid velocity profile). This is an interesting observation, given the known fact that gas-liquid bubble moves at a higher velocity than the liquid film. There are two possible explanations for this phenomenon. It could be a measurement bias effect caused by the averaging procedure due to variations on the stochastic position of the gas-liquid interface, or it could be caused by the fact that the interface is translating at a lower velocity than the gas-bubble. The no-slip condition at the gas-liquid interface would, therefore, decrease the velocity at the upper part of the liquid film.

To help answering this question, a different averaging procedure of the velocity profiles was implemented. This one was not based on the averaged velocity fields and the mean mask, but on the average of instantaneous velocity profiles obtained from the 1500 masked velocity fields. To account for variations on 

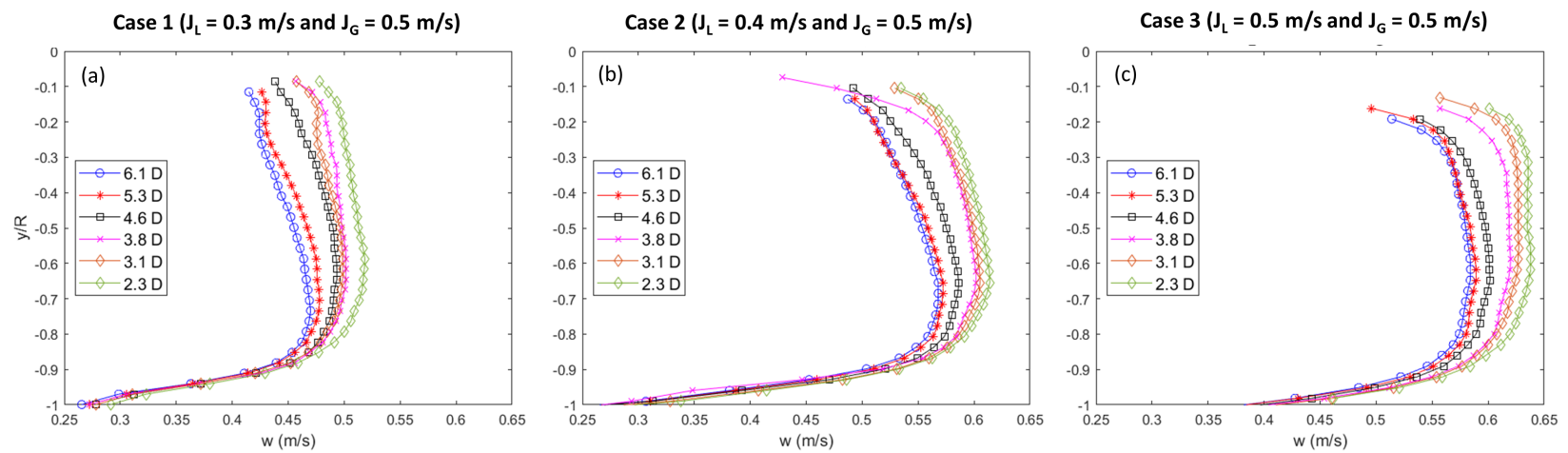

Figure 8: Averaged streamwise velocity profiles inside the liquid film at different positions upstream of the bubble nose tip.

the instantaneous positions of the gas-liquid interface and liquid flow rates, the velocity profiles were non dimensionalized by the film height (distance between the gas-liquid interface and the pipe bottom wall) and by the maximum streamwise velocity. Figure 9 presents the non-dimensional velocity profiles for different cross-sectional positions in the liquid film. Not only the same reduction of the liquid velocity close to the interface is observed, but all velocity profiles appear to be represented by a single self-similar curve. The result seems to validate the hypothesis that the gas-liquid interface translates at a smaller velocity than the gas bubble. Further investigation of the gas flow is needed to confirm this finding.
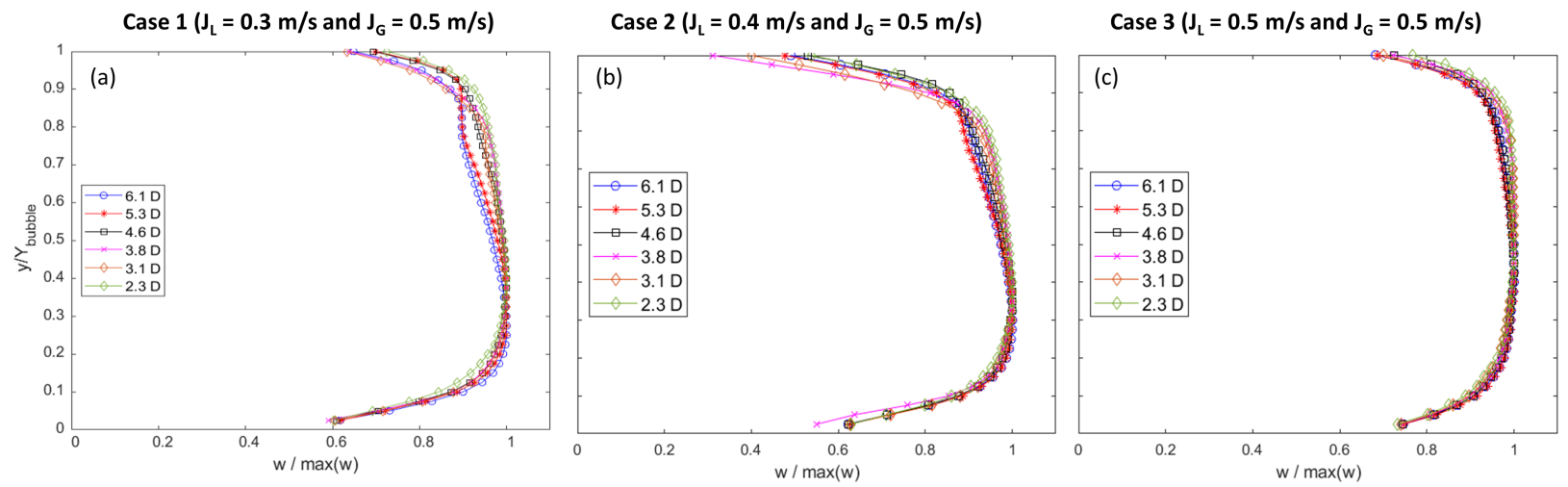

Figure 9: Self-similar vertical profiles of the averaged streamwise velocity, made non-dimensional by the interface height and the maximum streamwise velocity.

\section{Conclusions}

We analyzed, in the present work, averaged velocity fields and profiles at various cross-sectional positions for intermittent gas-liquid flows in a horizontal pipe using the gas-bubble nose tip as reference. To this end, a high-frequency stereoscopic-PIV system, together with the laser-induced fluorescence technique and photogate triggering were used.

For the liquid plug, it is clear that significant variations on the streamwise velocity profile occur up to $0.8 \mathrm{D}$ downstream of the gas-bubble nose, while at farther positions, the profile displays a shape similar to that of a fully-developed turbulent pipe flow. A slightly asymmetry on the velocity profile at the upper part of the pipe occurs due to the presence of dispersed bubbles.

The influence of the faster-moving gas-bubble on the velocity fields in the vicinity of the bubble nose tip was evaluated using mean algorithmic masks. The bubble nose pushes the downstream liquid forwards and downwards, forming a pair of counter-rotating streamwise vortices, in the liquid film. 
For the liquid film, the instantaneous masking procedure used allowed the determination of nondimensional profiles, that seem to converge into a single self-similar curve, independent of the axial distance from the gas-bubble nose tip. The velocity profiles obtained seem to indicate that the reduction observed in the velocity close to the gas-liquid interface is a physical phenomenon associated to the motion of the interface at a lower velocity than the gas bubble.

\section{Acknowledgements}

We would like to acknowledge the continuous financial support of Petrobras, CAPES an agency for the Brazilian Ministry of Education and CNPq, the Ministry of Science and Technology research council.

\section{References}

Al-Safran E, Sarica C, Zhang HQ, and Brill J (2005) Investigation of slug flow characteristics in the valley of a hilly-terrain pipeline. International journal of multiphase flow 31:337-357

Baker O (1953) Design of pipelines for the simultaneous flow of oil and gas. in Fall meeting of the petroleum branch of AIME. Society of Petroleum Engineers

Brennen CE (2005) Fundamentals of multiphase flow

De Oliveira WR, De Paula IB, Martins FJWA, Farias PSC, and Azevedo LFA (2015) Bubble characterization in horizontal air-water intermittent flow. International Journal of Multiphase Flow 69:18-30

Fabre J, Peresson LL, Corteville J, Odello R, and Bourgeois T (1990) Severe slugging in pipeline/riser systems. SPE Production Engineering 5:299-305

Fernandes LS, Martins FJWA, and Azevedo LFA (2018) A technique for measuring ensemble-averaged, three-component liquid velocity fields in two-phase, gas-liquid, intermittent pipe flows. Experiments in Fluids 59:1-18

Gonzales RC and Woods RE (2008) Digital image processing, 3rd ed

Hua G, Falcone G, Teodoriu C, and Morrison G (2012) Comparison of multiphase pumping technologies for subsea and downhole applications. Oil and Gas Facilities 1:36-46

Hurlburt E and Hanratty T (2002) Prediction of the transition from stratified to slug and plug flow for long pipes. International journal of multiphase flow 28:707-729

Lindken R and Merzkirch W (2002) A novel PIV technique for measurements in multiphase flows and its application to two-phase bubbly flows. Experiments in fluids 33:814-825

Taitel Y and Dukler AE (1976) A model for predicting flow regime transitions in horizontal and near horizontal gas-liquid flow. AIChE journal 22:47-55

Westerweel J and Scarano F (2005) Universal outlier detection for PIV data. Experiments in fluids 39:10961100

Wieneke B (2005) Stereo-PIV using self-calibration on particle images. Experiments in fluids 39:267-280

Woods BD and Hanratty TJ (1999) Influence of froude number on physical processes determining frequency of slugging in horizontal gas-liquid flows. International journal of multiphase flow 25:1195-1223 